\title{
Effect of Human Exogenous Leukocyte Interferon in Cytomegalovirus Infections
}

\author{
G. Emödi," R. O'Reilly, ${ }^{*}$ A. Müller, L. K. Everson, \\ U. Binswanger, $\dagger$ and M. Just
}

From the Department of Microbiology and Immunology, University Children's Hospital, Kantonsspital Basel,

Basel, Switzerland

\begin{abstract}
Human leukocyte interferon was injected into nine patients with cytomegalovirus infections; four of these patients were congenitally infected, and five had acquired infections. In three patients viruria was completely inhibited. In five patients viral excretion in the urine was only transiently inhibited. Viremia was not significantly suppressed. The lymphocyte response to phytohemagglutinin was suppressed in two patients.
\end{abstract}

Cytomegalovirus (CMV) has been associated with long-term, chronic infections in humans and causes the most common type of congenital infection. CMV has the ability to cross the placenta and to produce chronic infection in the fetus; such infection leads to serious pathologic changes or death. About $1.5 \%$ of all newborns excreting CMV in the urine after birth in one study [1] and approximately $3.3 \%$ of viruric newborns in other studies $[2,3]$ have had the classical signs of congenital CMV infection. Disseminated infection with CMV occurs in adults who have malignancies or who are taking corticosteroids [4], in children with leukemia [5, 6] or lymphosarcoma [7], and in patients who have had renal or bone-marrow transplantation $[8,9]$.

There is no established treatment for congenital or disseminated infection with cytomegalovirus. Only a few studies employing adequate virological monitoring of antiviral chemotherapy have been reported, and these studies revealed variable results in a small number of patients. Fluorodeoxyuridine, iododeoxyuridine, or cytosine arabinoside did not significantly alter the clinical course of illness or viral excretion [10-12].

Evidence exists that interferon may be one de-

This study was supported by grant no. 3.257 .69 from the "Schweizerischer Nationalfonds."

Please address requests for reprints to Dr. G. Emödi, Memorial Sloan Kettering Cancer Center, New York, New York 10012.

* Present address: Memorial Sloan Kettering Cancer Center, New York, New York 10012.

$\dagger$ Present address: Department of Medicine, Kantonsspital Zurich, Zurich, Switzerland. terminant of host resistance to viral infections [13-15]. In a nonrandomized study we have observed a possible antiviral effect of exogenous human leukocyte interferon in the treatment of local and generalized infections with herpes simplex virus and varicella-zoster virus $[15,16]$. Falcoff has reported a beneficial effect of exogenous amniotic interferon in the treatment of congenital CMV infection [17]. Our study was initiated to examine the effect of human leukocyte interferon on the rate of excretion of CMV in the urine and in fractions of the buffy coat of patients who are chronic carriers of CMV.

\section{Materials and Methods}

Human leukocyte interferon was prepared in our laboratory. Details regarding production, purification, standardization, and sterilization of this preparation have been described previously [15]. Interferon levels in administered preparations were assayed by microtitration techniques; vesicular stomatitis virus was inhibited in human foreskin fibroblast monolayers [18].

Isolations of virus from urine, leukocytes, and lymphocytes were based on growth and cytopathological characteristics. Clean urine was collected daily for a period of at least 20 days and processed immediately. Each of five tubes of confluent cultures of WI-38 human fibroblasts in Eagle's minimal essential medium (MEM) containing $2 \%$ inactivated fetal calf serum was inoculated with 0.4 $\mathrm{ml}$ of Millipore-filtered urine at different dilutions. Tubes containing monolayers that demonstrated CPE were subcultured, and CMV was identified 
by specific CPE in WI-38 cell cultures, presence of intranuclear inclusion bodies, lack of CPE in mouse L-cell cultures, and immunofluorescence [19]. For studies of antibody, CF tests were done by a micromethod [20].

Purified lymphocytes were obtained as previously described [21]. The leukocytes were prepared in the following manner. Heparinized blood $(10 \mathrm{ml})$ was allowed to sediment at $37 \mathrm{C}$ for 1-1.5 hr. Plasma rich in white blood cells (WBC) was collected and centrifuged at $300 \mathrm{~g}$ for $10 \mathrm{~min}$. The cells were washed three times in Hanks' balanced salt solution, resuspended in MEM with $2 \%$ fetal calf serum, and inoculated into WI-38 cells.

Lymphocyte responses to phytohemagglutinin (PHA) were tested in microculture [22]; the thymus-derived ( $\mathrm{T}$-)lymphocyte subpopulation was detected by the spontaneous rosettes that were formed with sheep erythrocytes. Surface immunoglobulins were tested according to the methods of Siegel et al. [23].

\section{Results}

Case reports. Patient no. 1 was a one-andone-half-year-old boy with congenital CMV infection (figure 1); he had had frequently recurring attacks of bronchial pneumonia and viral excretion since birth. His CF antibody titer was 1:60. Before treatment with human leukocyte interferon, he had a chronic cough with fever. Human leukocyte interferon was injected $\mathrm{im}$ in a single daily dose of $10^{6}$ units for 10 days. Before administration of interferon, all urine samples tested contained CMV. However, three days after commencement of treatment, no virus was detectable in the urine. Results of urine cultures were still negative six months after completion of treatment.

Patient no. 2 was a premature infant who had congenital CMV infection and hepatomegaly with minimal cerebral dysfunction (figure 1). Before treatment with human leukocyte interferon, all results of urine cultures tested for CMV were positive. The titer of virus was about $10^{2}-10^{3}$ $\mathrm{TCID}_{50} / 0.4 \mathrm{ml}$ of urine. A few leukocyte cultures were tested, and CMV was isolated from two of these cultures. The CF antibody titer of this patient was $1: 60$.

The patient was treated for eight days with a single daily dose of $5 \times 10^{5}$ units of human leukocyte interferon. No CMV was detectable in the urine after the fourth day of treatment. Results of subsequent urine cultures on days $30,62,68$, and 92 were negative. We were not able to isolate CMV from the only leukocyte culture tested.

Patient no. 3, a newborn weighing $2,000 \mathrm{~g}$, had congenital CMV infection and hepatosplenomegaly with severe thrombopenia (figure 2). During the first month of life, this patient had rectal bleeding and a thrombocyte count of $15,000 / \mathrm{mm}^{3}$. Before treatment with human leukocyte interferon, the patient's urine always contained $10^{2}-10^{3}$ $\mathrm{TCID}_{50}$ of $\mathrm{CMV} / 0.4 \mathrm{ml}$ of urine. Even purified lymphocyte fractions and leukocytes were positive for the virus. Human leukocyte interferon in single daily im doses of $10^{6}$ units was administered to attain a fairly high level of serum interferon with a duration of about $8 \mathrm{hr}$. Interferon was administered for 10 days. On day 4 of treatment, the patient's urine was free of virus. However, the inhibition was transient. Results of urine cultures were again positive on the 15 th day after the start of interferon administration and continued to

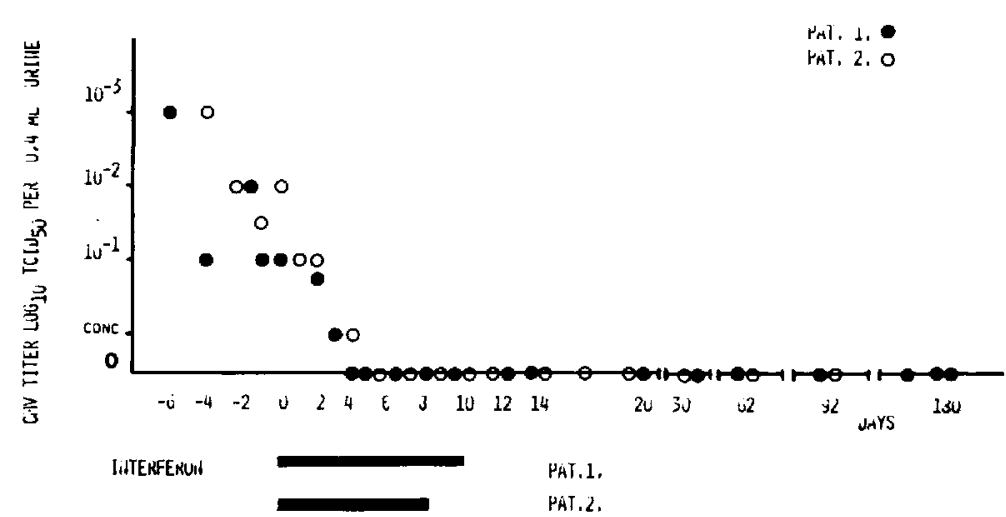

Figure 1. Excretion of cytomegalovirus (CMV) in the urine of patients no. 1 and no. 2 before, during, and after treatment with human leukocyte interferon. Patient no. 1 received $10^{6}$ units of interferon daily, and patient no. 2 received $5 \times 10^{5}$ units daily. 
Figure 2. Excretion of cytomegalovirus (CMV) in the urine and phytohemagglutinin (PHA) response of patient no. 3 before, during, and after treatment with human leukocyte interferon in a daily dose of $10^{6}$ units.
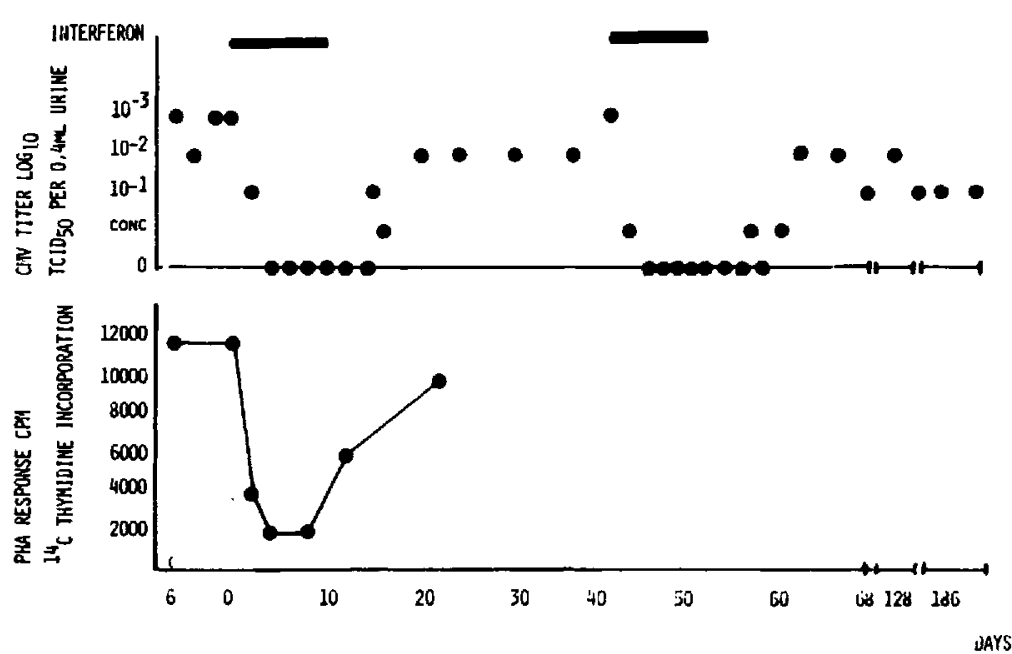

be positive until treatment was resumed on the same schedule and in the same dosage as used previously. Results of blood cultures were negative after the first course of treatment. The second course of treatment produced the same transient inhibition of virus in the urine as the first.

In this patient lymphocyte response to PHA was measured before, during, and after treatment. The PHA response was markedly suppressed as early as the second day of treatment. Five days after the last injection of interferon (first course), the PHA response returned, and, 22 days after the last injection, the PHA-stimulated cultures showed the same response as before administration, a finding that indicated normal T-cell function.

Patient no. 4 (figure 3 ) was a premature infant with a severe congenital CMV infection accompanied by cerebral dysfunction, motor retardation, hepatomegaly, and splenomegaly. During the first three months of life, he developed pneumonia. He continuously excreted a high titer of CMV in his urine. However, samples of buffy coat and plasma and lymphocyte cultures yielded no evidence of CMV. Lymphocyte cultures stimulated for interferon production showed a deficient production of circulating interferon. The patient's titer of CF antibody to CMV was 1:60.

This patient was treated for 10 days during three different periods with $2.5 \times 10^{5}$ units of human leukocyte interferon injected im once daily. Before the first treatment, all cultures of this patient's urine revealed $>10^{2} \mathrm{TCID}_{50}$ of CMV $/ 0.4 \mathrm{ml}$ of urine. Tests of daily urine cultures during treatment showed a possible transient reduction of viral excretion in the urine only in the first culture. This reduction was only marginal, from $10^{2}$ to $10^{1}$ $\mathrm{TCID}_{50}$. In the two later courses of treatment, no antiviral effect was observed, and the urine cultures had a constant titer of virus during the study.

Patient no. 5 was a 27 -year-old kidney transplant recipient who had received intensive immunosuppressive therapy and had experienced incipient rejection. The whole clinical course, with interstitial pneumonia, ascites, and increased rate of creatine clearance with rejection, was rapid. At the start of therapy with human leukocyte interferon, CPE was not detectable in the urine, blood, or peritoneal fluid. The only indication of CMV infection was a high titer $(1: 120)$ of CF antibody. Nevertheless, treatment with human leukocyte interferon was initiated in an im dose of $10^{6}$ units twice daily. The patient died on day 8 of treatment.

Results of all cultures taken before treatment, including urine, leukocytes, and macrophages isolated from the peritoneal fluid, were positive for CMV. CPE in the WI-38 cell culture was first observed after 25 days. A possible antiviral effect was observed in urine cultures after treatment for five days. In the last three days of treatment, urine cultures were negative for CMV, whereas leukocytes and macrophages isolated from the ascites had not been affected by the administration of interferon. An autopsy revealed typical CMV inclusion bodies in the kidney.

Patient no. 6, a 40-year-old man with an unusual type of CMV hepatitis, developed a generalized epidermolysis eight weeks after hepatitis 


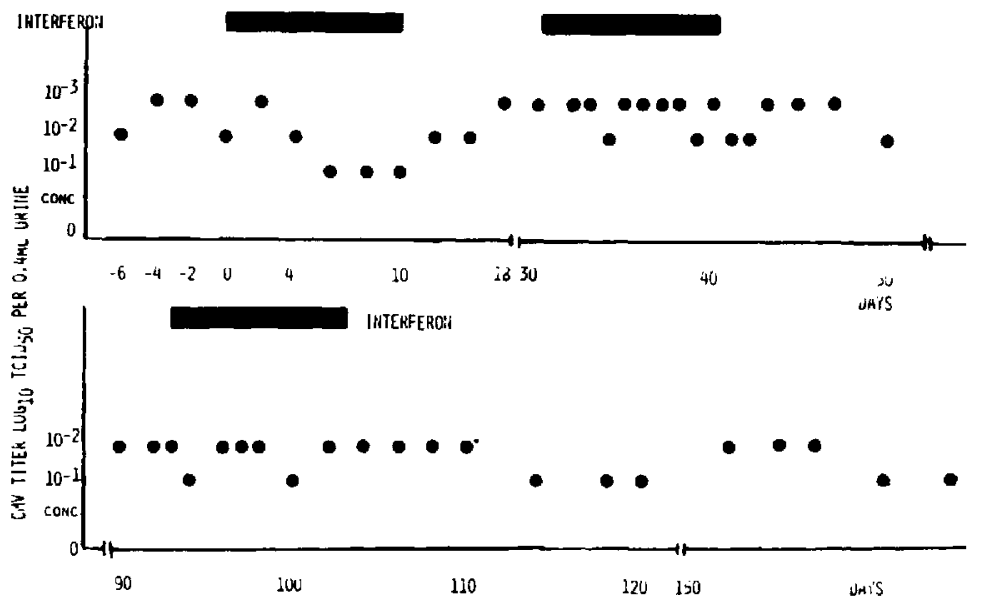

Figure 3. Excretion of cytomegalovirus (CMV) in the urine of patient no. 4 before, during, and after treatment with human leukocyte interferon (three courses; $2.5 \times 10^{5}$ units daily).

had been detected. Details of this case have been reported elsewhere [24]. During the hepatitis phase, he excreted virus in the urine, and leukocyte cultures also yielded CMV. After 10 days of treatment with human leukocyte interferon $\left(10^{6}\right.$ units daily, im), viral excretion in the urine was completely inhibited. No virus was isolated in 14 consecutive cultures during epidermolysis; however, CMV was isolated from the lymphocyte fraction.

Patient no. 7 was treated for herpes zoster with human leukocyte interferon. He had already developed an unproductive cough and increasing dyspnea when therapy was begun. CMV was isolated on the second day of treatment from his urine. He was treated im twice daily for 10 days with $10^{6}$ units of human leukocyte interferon. After six days viral excretion was inhibited, but only transiently. Urine cultures yielded CMV on days 15-18 and day 20 after initiation of treatment.

Two additional patients have been described previously. $^{\prime}$ Patient no. 8 was a six-month-old male with the adenosine deaminase deficiency form of severe combined immunodeficiency; he had had a bone-marrow transplantation. During transplantation his urine contained CMV. He was treated im for 10 days with $10^{6}$ units of human

${ }^{1}$ R. J. O'Reilly, L. K. Everson, G. Emödi, J. Hansen, E. Smithwick, E. Grimes, S. Pahwa, D. Armstrong, B. Dupont, and R. Good, "Effects of Exogenous Interferon in Cytomegalovirus Infections Complicating Bone Marrow Transplantation," presented at the Symposium on Antivirals with Clinical Potential, August 26-29, 1975 , Stanford, California. leukocyte interferon daily. Results of urine culture became only transiently negative for CMV. The PHA response was suppressed, as it was in patient no. 3.

Patient no. 9, a 24-year-old white female with acute myelogenous leukemia, had had a bonemarrow transplantation. She excreted CMV in the urine. She received a single injection of $5 \times 10^{6}$ units of human leukocyte interferon daily for 10 days. After treatment all urine samples tested yielded negative results. The last test was performed on day 92 with negative results.

Side effects. Human leukocyte interferon did not produce immediate or delayed serious side effects after im administration. Of the nine patients treated, two had local reactions, with redness and minimal tenderness at the site of administration. This local reaction lasted for about $12 \mathrm{hr}$. Fever reactions of $38 \mathrm{C}-38.5 \mathrm{C}$ were seen in two patients $6-8 \mathrm{hr}$ after administration, with a short duration of 4-6 hr.

\section{Discussion}

Infection with CMV in humans is associated with a long course of disease. Since congenital infection with CMV does not necessarily imply a bad prognosis, the possibility of antiviral treatment becomes important. Treatment is based on the unproved hypothesis that postnatal replication of CMV continues to damage the tissue of infants. Although unproved, the assumption that continued infection is damaging seems reasonable.

In acquired CMV infections in children and adults, the interaction between CMV and lympho- 
cytes may be important in the pathogenesis of the disease. In immunologically depressed patients, viruses might replicate in lymphocytes or in tissue, with autoreactive potential. In cases of immunological hyporesponsiveness induced by tumors, chemical agents, or conditioning therapy for transplantation [25], CMV might selectively replicate in an unrestricted fashion, culminating in a possibly fatal infection. In bone-marrow transplant recipients, the rather frequent graft-vs.-host disease is often accompanied by CMV infection. It is very difficult to distinguish symptoms of $\mathrm{CMV}$ infection from those of graft-vs.-host disease. Graft-vs.-host disease may be mediated by viruses. Hirsch et al. and De Maeyer et al. have described in their mouse studies not only an antiviral but also an anti-graft-vs.-host effect with a potent interferon preparation $[26,27]$.

In our investigation human leukocyte interferon had a long-lasting inhibitory effect on viral excretion of three of nine patients. A similar observation has been made by Arvin et al. [28]. It is not known whether the dosage used in this study was adequate; better results might be obtained with a larger dose or with twice daily administration of interferon, because the $t 1 / 2$ of interferon is $<24$ hr [29]. On the other hand, the immune response of the patients might be responsible for the results of therapy with human leukocyte interferon that were observed in treated patients. In addition, studies have shown that congenitally infected infants have a deficiency in production of circulating interferon [30].

The side effects observed during treatment with human leukocyte interferon, including a small local reaction at the injection site and fever, are of minor importance. These side effects can in no way be compared with those of chemotherapeutic agents such as fluorodeoxyuridine, iododeoxyuridine, or cytosine arabinoside. Long-term therapy with human leukocyte interferon may be possible without any serious side effects.

The immunosuppressive effects of interferon are well documented in vitro and in vivo (in animal models). Interferon suppresses the functions of T-cells, such as lymphocyte response to mitogens [31]. Our findings suggest that human leukocyte interferon may be useful in the treatment of CMV infections and may completely or transiently inhibit and/or partially reduce viral excretion in the urine and in the leukocytes. More observation is necessary to evaluate the usefulness of human leukocyte interferon in treatment of patients infected with CMV.

\section{References}

1. Birnbaum, G., Lynch, J. I., Margileth, A. M., Lonergan, W. M., Sever, J. L. Cytomegalovirus infections in newborn infants. J. Pediatr. 75:789, 1969.

2. Hanshaw, J. B. Congenital and acquired cytomegalovirus infection. Pediatr. Clin. North Am. 13:279, 1966.

3. McCracken, G. H., Shinefield, H. R., Cobb, K., Rausen, A. R., Dische, M. R., Eichenwald, H. F. Congenital cytomegalic inclusion disease. A longitudinal study of 20 patients. Am. J. Dis. Child. 117:522, 1969.

4. Rosen, P., Hajdu, S. Cytomegalovirus inclusion disease at autopsy of patients with cancer. Am. J. Clin. Pathol. 55:749-756, 1971.

5. Rinker, C. T., McGraw, J. P. Cytomegalic inclusion disease in childhood leukemia. Cancer 20:36-39, 1967.

6. Henson, D., Siegel, S. E., Fuccillo, D. A. Cytomegalovirus infections during acute childhood leukemia. J. Infect. Dis. 126:469-481, 1972.

7. Gottman, A. W., Beatty, E. C. Cytomegalic inclusion disease in children with leukemia or lymphosarcoma. Am. J. Dis. Child. 104:180-184, 1962.

8. Rifkind, D., Starzl, T. E., Marchioro, T. L. Transplantation pneumonia. J.A.M.A. 189:808-812, 1965.

9. Meyers, J. D., Spencer, H. C., Watts, J. C., Gregg, M. B., Stewart, J. A., Troupin, R. H., Thomas, E. D. Cytomegalovirus pneumonia after human marrow transplantation. Ann. Intern. Med. 82:181$188,1975$.

10. Feigin, R. D., Shackelford, P. G., De Vivo, D. C., Haymond, M. W. Floxuridine treatment of congenital cytomegalic inclusion disease. Pediatrics 48:318-319, 1971.

11. Conchie, A. F., Barton, B. W., Tobin, J. O. H. Congenital cytomegalovirus infection treated with idoxyuridine. Br. Med. J. 4:162, 1968.

12. Emödi, G., Sartorius, J., Just, M., Rohner, F., Bühler, U. Virus studies in the treatment of congenital cytomegalovirus infections by cytosine arabinoside. Helv. Paediatr. Acta 27:557-564, 1972.

13. Glasgow, L. A., Hanshaw, J. B., Merigan, T. C., Petralli, J. K. Interferon and cytomegalovirus in vivo and in vitro. Proc. Soc. Exp. Biol, Med. 125: 843-849, 1967.

14. Merigan, T. C. Clinical testing of human interferon in infectious disease. The production and use of interferon for the treatment and prevention of human virus infections. Monograph no. 3. Tissue Culture Association, Rockville, Md., 1975, p. 57. 
15. Emödi, G., Rufli, T., Just, M., Hernandez, R. M. Human interferon therapy for herpes zoster in adults. Scand. J. Infect. Dis. 7:1-5, 1975.

16. Kobza, K, Emödi, G., Just, M., Hilti, E., Leuenberger, A., Binswanger, U., Thiel, G., Brunner, F. P. Treatment of herpes infection with human exogenous interferon. Lancet 1:1343-1344, 1975

17. Falcoff, E., Falcoff, R., Fournier, F. Production en mass, purification partielle et characterisation d'un interferon destine des essais therapeutiques humains. Ann. Inst. Pasteur (Paris) 111:562-584, 1966.

18. Havell, E. A., Vilcek, J. Production of high-titered interferon in cultures of human diploid cells. Antimicrob. Agents Chemother. 2:476-484, 1972.

19. Schmitz, H., Haas, R. Erfahrungen mit der fluoreszenz-serologischen bestimmung von antikörpern gegen cytomegalievirus. Z. Med. Mikrobiol. Immunol. 155:283-287, 1970.

20. Hanshaw, J. B. Cytomegalovirus complement-fixing antibody in microcephaly. N. Engl. J. Med. 275: 476-479, 1966

21. Emödi, G., Just, M. Interferon production by lymphocytes in human milk. Scand. J. Immunol. 3: 157-160, 1974.

22. Du Bois, R., Meinesz, A., Bierhorst-Eijlander, A., Groenewoud, M., Schellekens, R. T., Eijsvoogel, V. P. The use of microtiter plates in mixed lymphocyte cultures. Tissue Antigens 4:456-468, 1972.

23. Siegel, F. P., Pernis, B., Kunkel, H. G. Lymphocytes in human immunodeficiency stages: a study of membrane-associated immunoglobulins. Eur. J. Immunol. 1:482-486, 1971.

24. Müller-Stamou, A., Senn, H. J., Emödi, G. Epidermolysis in a case of severe cytomegalovirus infection. Br. Med. J. 3:609-610, 1974.

25. Neuman, P. Interstitial pneumonia and cytomegalovirus infection as complications of human marrow transplantation. Transplantation 15:478-483, 1973.

26. Hirsch, M. S., Ellis, D. A., Profitt, M. R., Black, P. H., Chirigos, M. A. Effects of interferon on leukemia virus activation in graft versus host disease. Nature 244:10-103, 1973.

27. DeMaeyer, E., Mobraaten, L., DeMaeyer-Guignard, J. Prolongation par l'interferon de la servie des gryjes de peau chez la souris. C. R. Acad. Sci. (Paris) 277:2101-2103, 1973.

28. Arvin, A., Yeager, A., Merigan, T. C. Effect of leukocyte interferon on urinary excretion of cytomegalovirus by infants. J. Infect. Dis. 133(Suppl.): A205-A210, 1976.

29. Emödi, G., Just, M., Hernandez, R., Hirt, H. R. Circulating interferon in man after administration of exogenous human leukocyte interferon. J. Natl. Cancer Inst. 54:1045-1049, 1975.

30. Emödi, G., Just, M. Impaired interferon response of children with congenital cytomegalovirus disease. Acta Paediatr. Scand. 63:183-187, 1974.

31. Lindahl-Magnusson, P., Leary, P., Gresser, I. Interferon inhibits DNA synthesis induced in mouse lymphocyte suspensions by phytohaemagglutinin or by allogenic cells. Nature 237:120-121, 1972. 